\title{
Dual function of Arabidopsis ATAF1 in abiotic and biotic stress responses
}

Yaorong $\mathrm{Wu}^{1, *}$, Zhiyong Deng ${ }^{2,3, *}$, Jianbin Lai ${ }^{1,2}$, Yiyue Zhang ${ }^{1,2}$, Cuiping Yang ${ }^{2}$, Bojiao Yin ${ }^{1,2}$, Qingzhen Zhao , Ling Zhang ${ }^{2}$, Yin Li ${ }^{2}$, Chengwei Yang ${ }^{2}$, Qi Xie ${ }^{1,2}$

${ }^{I}$ State Key Laboratory of Plant Genomics, National Center for Plant Gene Research, Institute of Genetics and Developmental Biology, Chinese Academy of Sciences, Datun Road, Beijing 100101, China; ${ }^{2}$ State Key Laboratory for Biocontrol, School of Life Science, Sun Yat-sen (Zhongshan) University, Guangzhou 510275, China

NAC family genes encode plant-specific transcription factors involved in diverse biological processes. In this study, the Arabidopsis NAC gene ATAF1 was found to be induced by drought, high-salinity, abscisic acid (ABA), methyl jasmonate, mechanical wounding, and Botrytis cinerea infection. Significant induction of ATAF1 was found in an ABA-deficient mutant aba2 subjected to drought or high salinity, revealing an ABA-independent mechanism of expression. Arabidopsis ATAF1-overexpression lines displayed many altered phenotypes, including dwarfism and short primary roots. Furthermore, in vivo experiments indicate that $A T A F 1$ is a bona fide regulator modulating plant responses to many abiotic stresses and necrotrophic-pathogen infection. Overexpression of ATAF1 in Arabidopsis increased plant sensitivity to ABA, salt, and oxidative stresses. Especially, ATAF1 overexpression plants, but not mutant lines, showed remarkably enhanced plant tolerance to drought. Additionally, ATAF1 overexpression enhanced plant susceptibility to the necrotrophic pathogen $B$. cinerea, but did not alter disease symptoms caused by avirulent or virulent strains of $P$. syringae pv tomato DC3000. Transgenic plants overexpressing ATAF1 were hypersensitive to oxidative stress, suggesting that reactive oxygen intermediates may be related to $A T A F 1$-mediated signaling in response to both pathogen and abiotic stresses.

Keywords: abiotic stress, Arabidopsis, ATAF1, biotic stress

Cell Research 19:1279-1290. doi: 10.1038/cr.2009.108; published online 15 September 2009

\section{Introduction}

Plants are continuously exposed to various abiotic and biotic stresses in natural environment, and have evolved many adaptive mechanisms, which are dependent on a variety of signaling pathways, in order to react to altered conditions and combat pathogen attacks for survival. These stress-related signaling pathways are individually regulated by phytohormones including abscisic acid (ABA), salicylic acid (SA), jasmonic acid (JA),

\footnotetext{
* These two authors contributed equally to this work.

${ }^{3}$ Current address: Wake Forest University School of Medicine, WinstonSalem, NC 27157, USA

Correspondence: Qi Xie

Tel: 86-10-64889351; Fax: 86-10-64889351

E-mail: qxie@genetics.ac.cn

Received 11 February 2009; revised 16 April 2009; accepted 8 May 2009; published online 15 September 2009
}

and ethylene (ET) [1-4], and can result in various physiological responses such as osmotic adjustment, stomatal movement, and oxygen bursts. The cellular processes of plant-stress responses are further complicated by webtype crosstalks among different signaling pathways. The identification and characterization of important signaling components have provided insights into these specific pathways; however, gaps still exist in our understanding of plant-stress response mechanisms. Transcriptome analyses have generated considerable data, which show extensive overlapping on gene expression between biotic and abiotic stresses. Many of these overlapped genes encode signaling components, including transcription factors and protein kinases [5-9].

Transcription factors are promising candidates of common players involved in crosstalk between abiotic and biotic stress signaling [4]. For instance, AtMYC2/JIN1, encoding the basic helix-loop-helix transcription factor, is not only involved in the ABA-mediated drought stress- 
signaling pathway, but also involved in JA-mediated wound response and JA/ET-mediated pathogen defense responses. Overexpression of AtMYC2 resulted in improved osmotic stress tolerance. AtMYC2 deficiency resulted in elevated expression of JA/ET-regulated defense genes and increased plant resistance to the necrotrophic fungal pathogen Fusarium oxysporum [10-12]. Additionally, BOS1, an R2R3MYB transcription factor, was shown to be required for biotic and abiotic stress responses in Arabidopsis. bos 1 mutant plants showed increased susceptibility to necrotrophic pathogens, and impaired tolerance to drought, salinity, and oxidative stress [13].

Many transcription factors involved in signaling crosstalk are multi-functional proteins. As a large multifunctional gene family, the Arabidopsis NAC family contains more than one hundred members. NAC proteins are plant-specific transcription factors characterized by a conserved N-terminal region known as the NAC domain. NAC proteins have crucial functions in plant developmental processes. Several NAC members were found to be determinative regulators in diverse plant developmental programs, including formation of apical shoots [14], lateral roots, and floral organs [15-17]; and leaf senescence [18]. Additionally, NAC proteins have been implicated in disease defense and abiotic stress signaling. Several NAC proteins have been shown to interact with virus proteins [19-21], or to play direct roles in regulating pathogen defense [22]. Some NAC proteins were recently identified as abiotic stress regulators. The NAC cDNAs ANAC019, ANAC055, and ANAC072/RD26 were isolated as promoter-binding proteins of ERD1 (early-responsive to dehydration 1). Their expression was induced by drought, high salinity, ABA, and methyl jasmonate (MeJA). Plants individually overexpressing these genes showed a significant increase in drought tolerance or salt tolerance [23-26]. The anac019anac055 double mutant showed increased resistance to $B$. cinerea compared with the wild-type plants, and transgenic plants overexpressing ANAC019 or ANAC055 showed decreased resistance to this pathogen [27]. Moreover, NAC transcription factors such as ATAF2 and OsNAC6 have been shown to be involved in both biotic and abiotic stress responses. Overexpression of the ATAF2 gene, which is a wound-, SA-, JA-, and salinity-responsive gene, resulted in increased susceptibility to fungal pathogens and decreased expression of pathogenesis-related genes [28]. Additionally, the rice gene OsNAC6 was found to be an abiotic and biotic stress-related gene. OSNAC6-overexpression plants were tolerant to dehydration/high-salinity stresses and showed slightly increased tolerance to blast disease $[29,30]$.

ATAF1 (At1g01720), encoding a NAC protein, was previously reported to be a wounding-inducible gene [19]. The ataf1-1 mutant line showed decreased resistance to a biotrophic fungus as a consequence of increased ABA level $[22,31]$. ATAF1 was also reported to be a negative regulator in drought response based on analysis of ataf1 mutants [32]. Here, we show that ATAF1 plays important roles in both abiotic and biotic stress responses. We found that ATAF1 overexpression lines, but not its null mutants, showed enhanced plant drought tolerance, revealing a positive function in plant drought response. Additionally, ATAF1 overexpression lines were hypersensitive to high salinity, ABA, oxidative stress, and necrotrophic-pathogen infection. Our data indicate that ATAF1 mediates Arabidopsis response to both abiotic and biotic stresses.

\section{Results}

\section{Arabidopsis ATAF1 is a potential stress-related NAC} gene

In order to systematically survey the function of Arabidopsis NAC members in stress signaling, we conducted an in silico analysis by mining public microarray data (Arabidopsis Biological Resource Center) for all NAC family genes under different stress treatments. As summarized in Supplementary information, Table S1, members of NAC family genes were found to be induced by multiple stresses, suggesting functional redundancy as well as specificities. We are in the process of applying forward and reverse genetic approaches to functionally study these stress-related NAC members. The following experimental results are based on our detailed investigation of one stress-related $N A C$ representative, ATAF 1 (At1g01720, ANAC002).

\section{Upregulation of ATAF1 in response to multiple abiotic} stresses and Botrytis cinerea infection

To confirm the microarray data, detailed expression patterns of $A T A F 1$ were analyzed by RNA gel blot using an $A T A F 1$-specific probe. ATAF1 transcripts rapidly accumulated upon $250 \mathrm{mM} \mathrm{NaCl}$, dehydration, or $50 \mu \mathrm{M}$ ABA, and were maintained at a high level during the entire treatment period. Furthermore, ATAF1 was found to be upregulated by mechanical wounding and $50 \mu \mathrm{M}$ MeJA. In addition to induction of ATAF1 by these abiotic stimuli or stress-related phytohormones, accumulation of ATAF 1 transcripts was also observed in seedlings infected with $B$. cinerea (Botrytis cinerea) (Figure 1). ABA is generally considered to be an important biomolecule regulating plant responses to drought and high salinity, and in particular it plays an essential role in some ABA-dependent stress response processes. To determine 

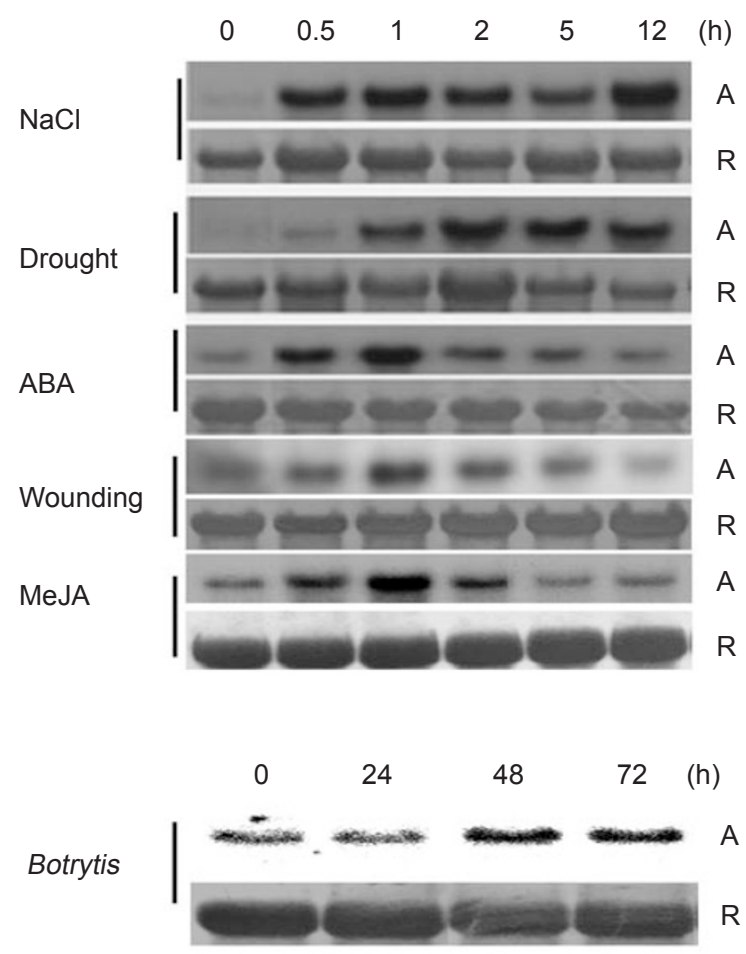

Figure 1 Northern blot analysis of ATAF1 transcript. Expression of ATAF1 in response to high salinity ( $250 \mathrm{mM} \mathrm{NaCl}$ ), drought, ABA $(50 \mu \mathrm{M})$, wound, MeJA $(50 \mu \mathrm{M})$ treatments, and $B$. cinerea infection. A: ATAF1 probe. R: rRNA: methylene blue-stained ribosome RNA used as loading controls.

whether ATAF1 accumulation during drought and salinity stresses was dependent on ABA, aba2 (an Arabidopsis ABA-deficient mutant) was used for analyzing ATAF1 gene expression. The RNA gel blot revealed that ATAF1 was still evidently induced in aba2 plants subjected to drought or $\mathrm{NaCl}$ treatment (Supplementary information, Figure S1). This finding indicates that induction of ATAF1 in response to drought or high salinity can be achieved through an ABA-independent pathway, although ABA alone is able to induce ATAF1 expression.

\section{Genetic analysis of ATAF1 gene}

To investigate the functions of ATAF 1 in vivo, we generated transgenic Arabidopsis plants overexpressing this gene. RNA gel blot revealed that all of the transgenic lines expressed ATAF1 at higher levels compared with wild-type plants (data not shown). The highest level of $A T A F 1$ expression was found in three transgenic lines, designated as ATAF1-O1, ATAF1-O2, and ATAF1-O3. In western blot assays, these three lines exhibited high expression of the Myc-ATAF1 protein (Figure 2A). In addition, two T-DNA insertion lines with different target sites in ATAF1 gene exons were obtained from the Salk mu- tant collection: Salk-067648 (ataf1-1) and Salk-057618 (ataf1-2). RT-PCR results showed that the expression of ATAF1 was completely abolished in the atafl-1 and ataf1-2 mutant lines, but was higher in the overexpressing lines (Figure 2B).

The ATAF1-transgenic lines showed altered phenotypes, such as relative dwarfism with small size, light green and round-shaped leaves, short petioles, sterility at the early flowering stage, and short siliques. Although ataf1 mutants exhibited long primary roots (data not shown), their phenotypes bear a close resemblance to those of wild-type plants (Figure 2C).

A

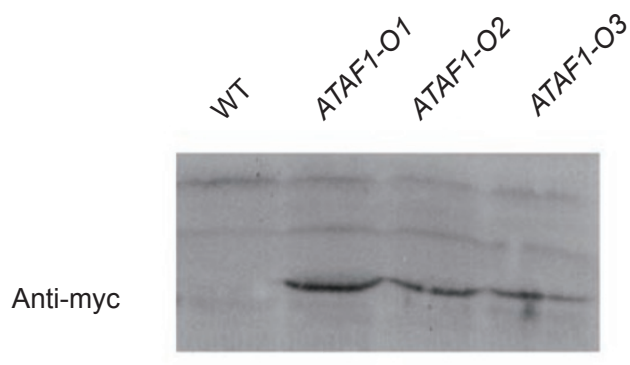

B

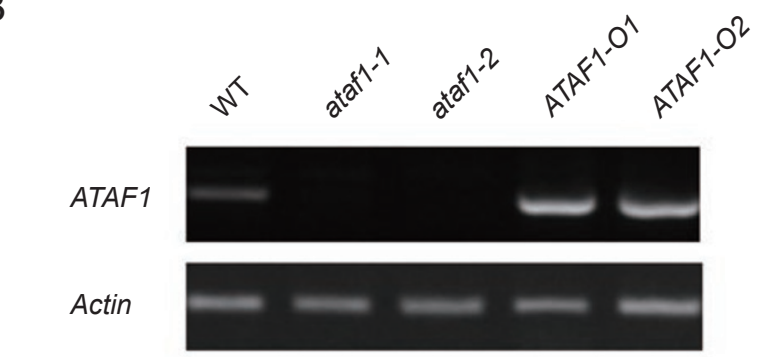

C WT ataf1-1 ataf1-2 ATAF1-O1 ATAF1-O2

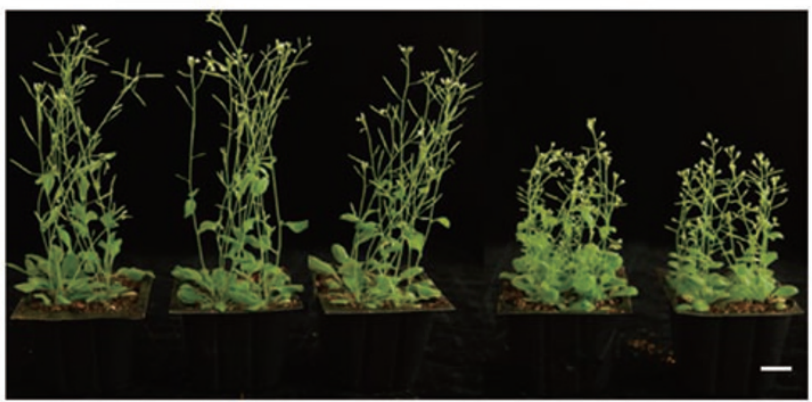

Figure 2 Genetic analysis of ATAF1. (A) ATAF1 transcript and protein levels in ATAF1-overexpression lines. Western blot was performed with anti-Myc monoclonal antibody. (B) Comparison of ATAF1 transcript levels in wild-type, ataf1-1, ataf1-2, overexpression plants ATAF1-O1 and ATAF1-O2. (C) Growth phenotype of 35-day-old ATAF1-overexpression plants in comparison with ataf1 mutants and wild-type plants. WT: wild-type plants. Bar $=1 \mathrm{~cm}$. 
Overexpression of ATAF1 confers drought tolerance in Arabidopsis

Since $A T A F 1$ is a drought-inducible gene, it likely regulates drought signaling. To characterize such a function of $A T A F 1$, a whole-plant drought assay was performed in soil. Seedlings (10-day old) were transferred from MS plates to water-saturated soil for 7 days, and then water

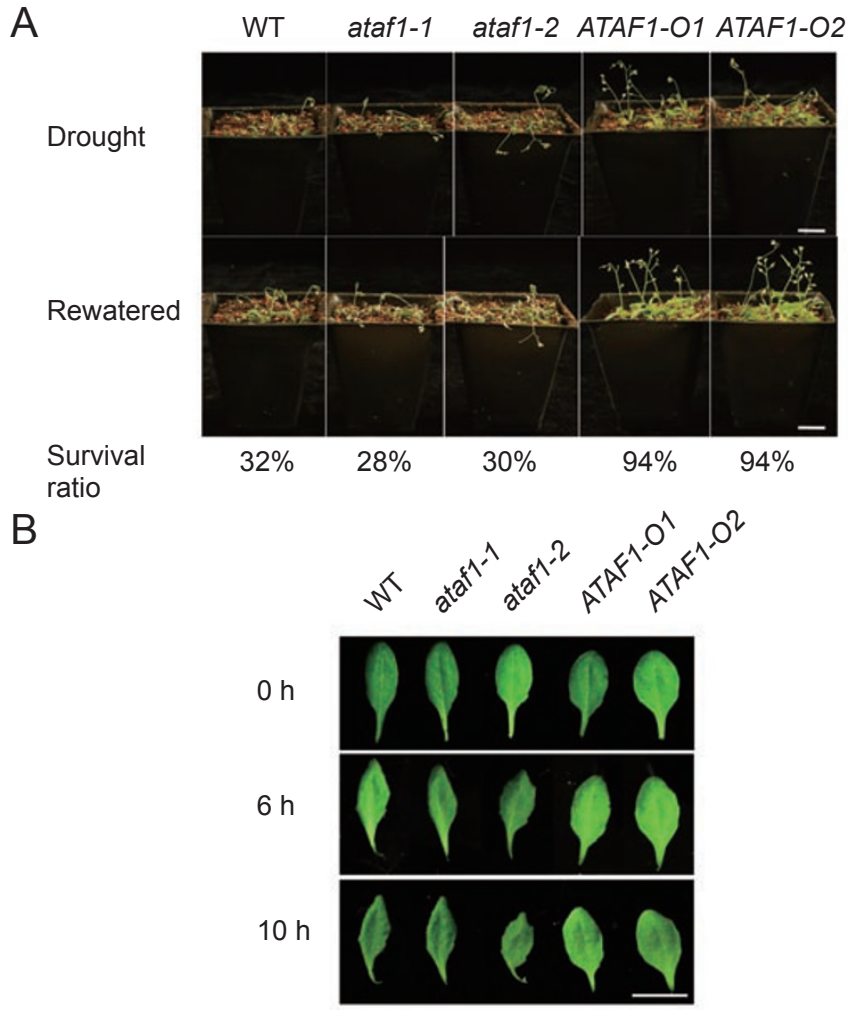

C

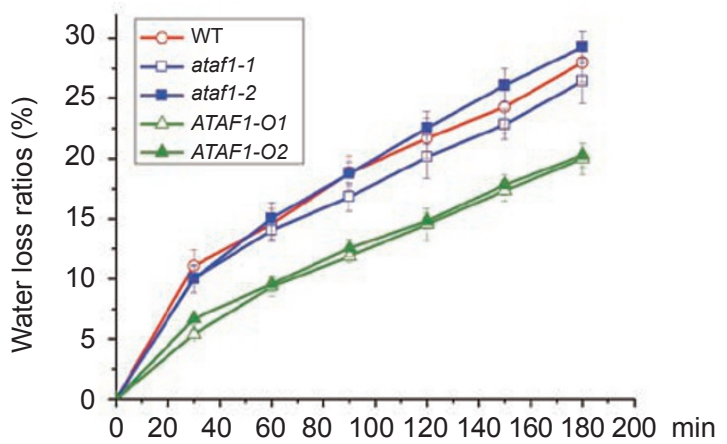

Figure 3 Drought-tolerance assays of wild-type, ataf1-1, ataf1-2, ATAF1-01, and ATAF1-O2 plants. (A) Photographs were taken when the drought-stressed phenotypes appeared and $6 \mathrm{~h}$ after being rewatered. Bar $=1 \mathrm{~cm}$. (B) Comparison of phenotypes of excised leaves under the air-dry treatment for $0 \mathrm{~h}, 6 \mathrm{~h}$, and $10 \mathrm{~h}$. Bar $=1 \mathrm{~cm}$. (C) Leaf water loss assay. Leaf weight was measured at the indicated time point $(n=18)$. Curves were drawn based on the data from three independent experiments, and bars indicate standard errors. was withheld to cause a severe water deficit in soil. After 21 days, most of the control and mutant plants were wilted due to the extreme water deprivation (Figure 3A). After rewatering, 94\% of overexpression plants continued to grow and successfully produced seeds, whereas most of the other plants did not recover and died from drought, showing a lower survival ratio (32\% for wildtype, $28 \%$ for ataf1-1, and 30\% for ataf1-2) (Figure 3A). This experiment was repeated several times with similar results. Our results indicate that high levels of ATAF1 can enhance plant drought tolerance.

Regulation of transpiration plays a vital role in plant response to drought stress. To address whether the ATAF1 function in enhancing plant drought tolerance was associated with transpiration, we compared the wilting phenotype of detached leaves from different plants that were subjected to air-dry treatment at room temperature with $65 \%$ humidity. After $6 \mathrm{~h}$, obvious phenotypic changes were observed in these leaves (Figure 3B). At this time point, only the leaves from ATAF1-O1 and ATAF1-O2 plants retained normal shape and they only showed a slightly wilting phenotype, whereas the leaves of other plants were severely wilted. Similar phenomena were observed at $10 \mathrm{~h}$ of the treatment (Figure 3B). We further addressed the reasons for these phenotypic alterations by measuring water loss ratios in detached leaves from these plants (Figure $3 \mathrm{C}$ ). In this assay, we found that the leaf weight of ATAF1-O1 and ATAF1-O2 plants decreased at the slowest rate of all analyzed plants, whereas other plants showed comparable water loss rates. Therefore, this quantitative result is consistent with the phenotypic observations in the leaf-wilting assay.

To reveal how ATAF1 controls drought-responsive genes, we examined the expression of a group of marker genes in the drought-stressed plants (Supplementary information, Figure S2). ATAF1-O1 plants produced dramatically high basal levels of $A D H 1$ (encoding an alcohol dehydrogenase) transcripts $(0 \mathrm{~h})$ and further increased its expression to reach the highest level compared with other plants. The expression patterns of RD29A and COR 47 were very similar. They showed low and comparable basal expression among different plants at $0 \mathrm{~h}$, and then divergently increased in the assayed drought-stressed plants. After $2 \mathrm{~h}$ of treatment, transcripts of these two markers accumulated to a higher level in ataf1-1 plants compared with wild-type or overexpression lines. However, transcript levels decreased in atafl-1 plants thereafter (10 vs 2 h). In contrast, ATAF1-O1 plants induced small amounts of transcripts of the two marker genes at the 2-h time point, but accumulated the highest level of their transcripts at late stages of stress $(10 \mathrm{~h})$ in comparison with the other assayed plants. The drought-induction 
of $R D 22$ was markedly repressed in ATAF1-O1 plants, but slightly enhanced in the mutant plants. These results indicate that ATAF1 may control different downstream ABA- and stress-responsive genes through specific man- ners that are different from other transcription factors.

Altered ATAF1 expression affects plant sensitivity to ABA The transcript level of $A T A F 1$ was dramatically in-
A

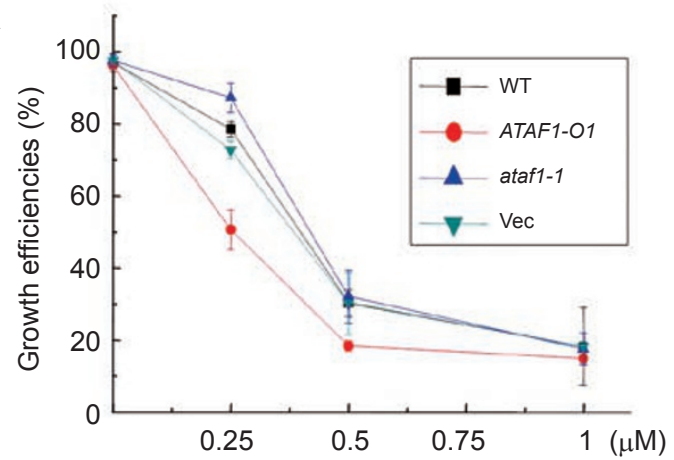

B
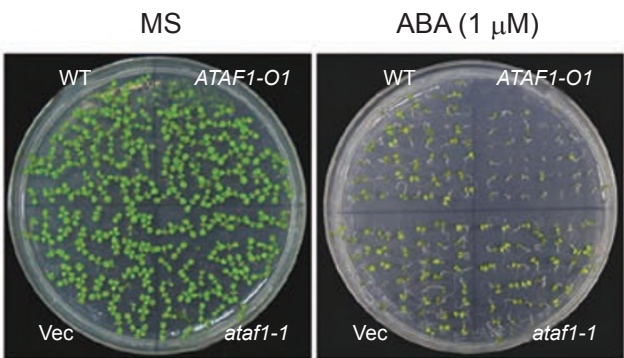

C

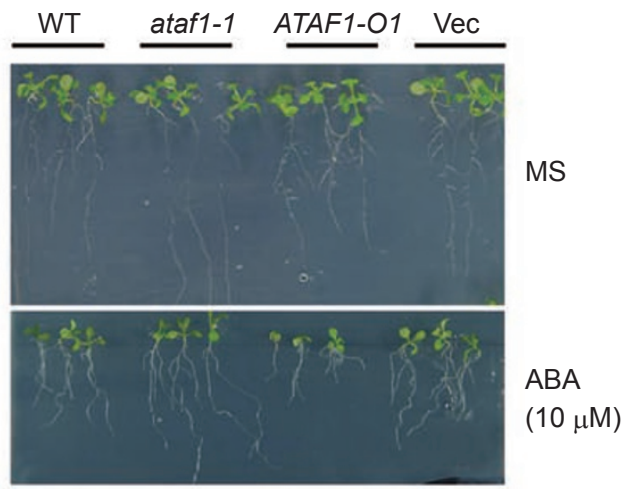

D

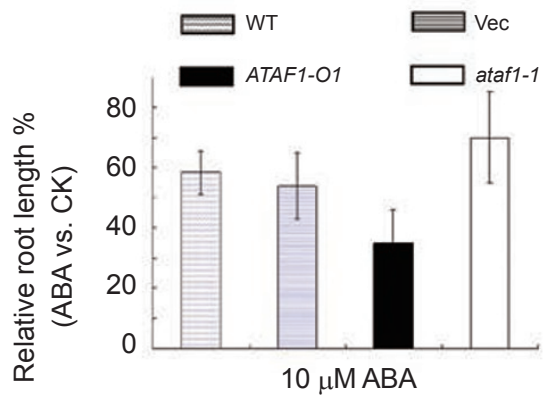

$\mathrm{F}$

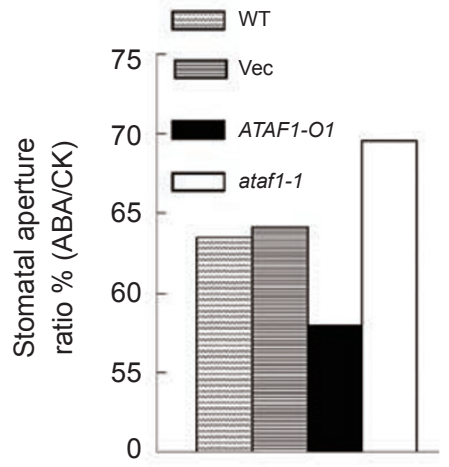

Figure 4 Sensitivity of ATAF1 overexpression lines to ABA. (A) Effects of ABA on growth efficiencies. Growth efficiencies (green cotyledons) represented by the chart were based on three repeated experiments, in each of which an average of 90 seeds were counted. (B) Seedlings were grown on MS medium with or without $1 \mu \mathrm{M} A B A$ and their phenotypes were scored on the 12th day after sowing. (C) Phenotypic comparison of root length in the presence of ABA. (D) Quantitative analysis of root growth. The experiment was performed in triplicate $(n=20)$ and repeated thrice. Bars indicate standard errors. (E) Effects of $\mathrm{ABA}$ on stomatal aperture of plants. Epidermal peels from plants were kept for $12 \mathrm{~h}$ in the dark, incubated under light in buffer for $3 \mathrm{~h}$, and then treated with 0 and $50 \mu \mathrm{M} \mathrm{ABA}$ for $2 \mathrm{~h}$ before aperture measurements. Data are mean ratios of width to length \pm SE of three independent experiments $(n=30$ to 40 ). WT: wild type, Vec: vector control plants. (F) Stomatal aperture ratio, data are ratios of stomatal aperture of plants treated with $50 \mu \mathrm{M} A B A$ to that of untreated plants. 
duced by ABA in RNA gel blot assay (Figure 1), suggesting the potential function of $A T A F 1$ in ABA response. To elucidate the role of ATAF1 in ABA signaling, ATAF1O1, ataf1-1 mutant, and control plants were germinated on MS plates with different amounts of $\operatorname{ABA}(0,0.25,0.5$, and $1 \mu \mathrm{M}$ ). No difference in the growth efficiencies (green cotyledons) and germination rates was observed among the untreated plants (Figure 4A). However, in the presence of $0.25 \mu \mathrm{M} \mathrm{ABA}$ for 3 days (the lowest concentration tested), the growth efficiencies decreased to $51.3 \%$ for ATAF1-O1, 78.5\% for wild-type plants, $72.8 \%$ for vector plants, and $87.2 \%$ for atafl-1 plants. Similar inhibition effects were also observed for these plants grown on $0.5 \mu \mathrm{M}$ ABA plates. ATAF1-O1 plants exhibited the most severe growth inhibition by ABA among the assayed plants, indicating that high levels of ATAF1 transcripts rendered plants more sensitive to ABA. The highest concentration of ABA $(1 \mu \mathrm{M})$ used in this study led to extreme growth inhibition for all of the plants (Figure 4A); however, only ATAF1-O1 plants displayed the most severely arrested growth under this condition, as reflected by retardation of cotyledon greening (Figure 4B). On the contrary, the germination rate of atafl-1 plants was the lowest and the germination rate of ATAF1-O1 was the highest compared with that of the control plants (data not shown). To further correlate ATAF1 function with plant sensitivity to ABA during the post-germination stage, root elongation inhibition was analyzed for these plants. Seedlings (4-day old) were transferred to MS medium with $10 \mu \mathrm{M}$ ABA. After 7 days, root growth inhibition by ABA was the least severe for the ataf1-1 mutant, but the most severe for ATAF1-O1 plants among the assayed plants (Figures 4C, 4D). Taken together, these results indicate that ATAF1 modulates plant ABA signaling and high ATAF1 expression contributes to ABA hypersensitivity in Arabidopsis.

Stomata numbers on the leaf and aperture of stomata are major factors to determinate water evaporation. Under microscope, stomata numbers in identical area were counted for ATAF1-O1, ataf1-1, and control plants, and no stomata number changes were observed (data not shown). Overexpression of some ABA signaling components, such as $S D I R 1, A B F 3$, and $A B F 4$, can reduce leaf water loss, because these genes have regulatory functions in controlling stomatal movement $[33,34]$. To determine whether ATAF1 can act on the adjustment of stomatal openings in a similar manner, we treated leaves of four genotypes with $\mathrm{ABA}$ to analyze stomatal aperture. Treating the leaf epidermis of ATAF1-O1 plants with ABA caused nearly complete closure of stomata; the effect was not so pronounced in the control and atafl-1 plants (Figure 4E). Indeed, treating the leaf epidermis of ATAF1-O1

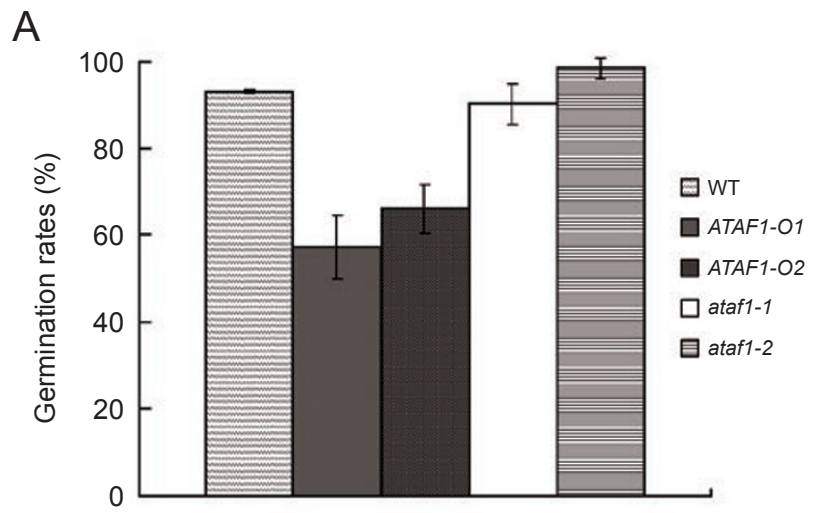

B
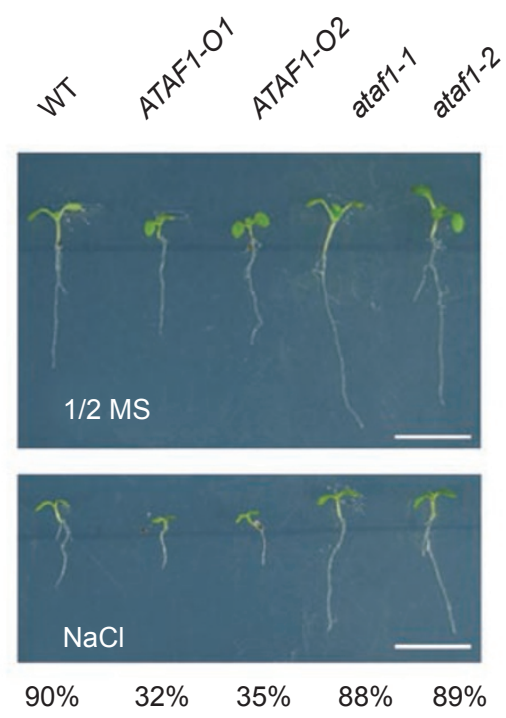

Figure 5 High-salinity assay of plants. (A) Germination rates (radical emergence) of wild type, ataf1-1, ataf1-2, ATAF1-01, and ATAF1-O2 plants on 1/2 MS medium containing $100 \mathrm{mM}$ $\mathrm{NaCl}$ for 5 days. Percentages are means ( $n=60$ to 90 each) of three repeats $\pm S E$. (B) Growth phenotype of transgenic and mutant plants on 1/2 MS medium (top panel) or containing 100 $\mathrm{mM} \mathrm{NaCl}$ (bottom panel). Seeds were germinated and grown for 7 days. Numbers below the panel indicate the percentage of seedlings with green cotyledons in total seedlings grown on $100 \mathrm{mM} \mathrm{NaCl}$ medium ( $n=60$ to 90 ). $\mathrm{Bar}=1 \mathrm{~cm}$.

plants with ABA caused the most severe closure of stomata $(57.9 \%$ of untreated stomata aperture) compared with other plants $(63.4 \%$ for wild-type plants, $64.2 \%$ for Vec control plants, and $69.5 \%$ for ataf1-1 plants) (Figure $4 \mathrm{~F})$. Obviously, ATAF1 overexpression elevated the sensitivity of stomatal closure mediated by ABA, whereas ataf1-1 mutants showed slightly opposite phenotypes. These results indicate that ATAF1 is involved in ABAdependent stomatal closure. 
To further substantiate this finding, the expression of several $\mathrm{ABA} /$ stress-responsive marker genes was analyzed in ATAF1-O1, ataf1-1, and wild-type plants (Supplementary information, Figure S3). For $A D H 1$ gene, wild-type and ataf1-1 plants showed similar expression patterns in response to ABA treatment. However, $A D H 1$ was highly expressed in ATAF1-O1 plants at the $0 \mathrm{~h}$ time point and its transcripts were further increased by $\mathrm{ABA}$ treatment. Differently, $R D 22$ showed higher induction by ABA in the ataf1-1 mutant than ATAF1-overexpression and wild-type plants (Supplementary information, Figure S3). The results suggest that ATAF1 is involved in plant response to $\mathrm{ABA}$ by differentially regulating $\mathrm{ABA} / \mathrm{stress}-$

A

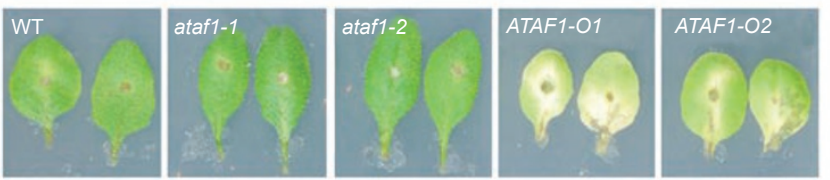

B

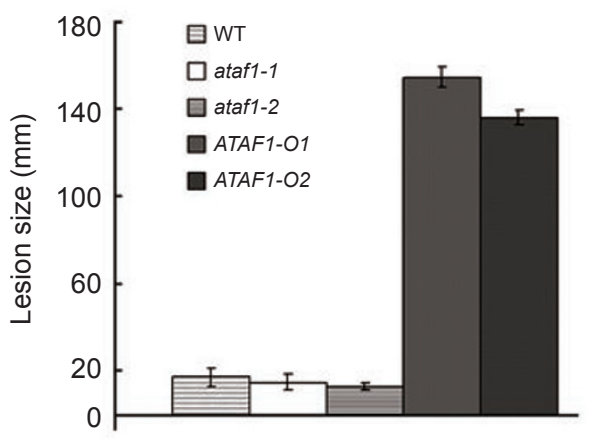

C

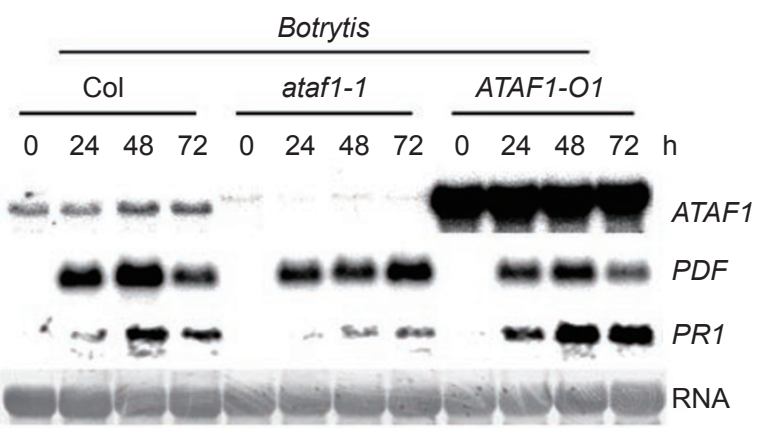

Figure 6 Responses of plants to $B$. cinerea infection. (A) Disease symptoms of detached leaves 5 days after inoculation with a $3-\mu l$ droplet of $B$. cinerea spores $\left(10^{5}\right.$ spores $\left./ \mathrm{ml}\right)$. (B) Lesion size was measured 5 days after inoculation. Date points represent average lesion size of measurements from 30 lesions. The experiments were repeated thrice. (C) Expression of $P R-1$ and $P D F 1.2$ in response to $B$. cinerea infection. Each lane contains $15 \mu \mathrm{g}$ total RNA. responsive genes.

Transgenic plants overexpressing ATAF1 are hypersensitive to high salinity

ATAF1 was found to be induced by salt; therefore, we asked whether this induction was correlated with plantsalinity response. As shown in Figure 5A, the germination rates (radicle emergence) of ATAF1-O1 and ATAF1$O 2$ were dramatically influenced by $100 \mathrm{mM} \mathrm{NaCl} \mathrm{com-}$ pared with those of other plants. There was no difference of germination rates among all untreated plants (data not shown). Moreover, the greatest growth retardation and lesion were also found for ATAF1-O1 and ATAF1-O2 in the presence of $100 \mathrm{mM} \mathrm{NaCl}$ (Figure 5B). The percent of green seedlings was $32 \%$ and $35 \%$ for ATAF1-O1 and ATAF1-O2, and nearly $90 \%$ for other plants (Figure 5B). Similar phenomena were also observed under a lower $\mathrm{NaCl}$ concentration $(50 \mathrm{mM})$ (data not shown). These experiments indicate that high level of ATAF1 transcripts sensitizes plants to high salinity.

To further address the regulatory function of ATAF1 in salt response, expression patterns were checked for stress-responsive marker genes under $\mathrm{NaCl}$ treatment. Similar to $\mathrm{ABA}, \mathrm{NaCl}$ induction led to high $A D H 1$ expression in ATAF1-O1 plants, but did not notably change its expression in the ataf1-1 mutant. Additionally, three downstream stress-signaling genes, RD22, RD29A, and COR47, showed similar expression patterns. Their expression, which was unaffected in the ataf1-1 mutant, was reduced in ATAF1-O1 plants at the early stage of salt stress $(2 \mathrm{~h})$. Interestingly, the transcripts of these three genes were slightly increased in ATAF1-O1 plants at the late treatment stage $(10 \mathrm{~h})$. These results may suggest a feedback regulation of those genes affected by ATAF 1 under salt stress conditions (Supplementary information, Figure S4).

Altered ATAF1 expression levels affect plant susceptibility to the fungal pathogen $B$. cinerea

ATAF1 has been shown to be induced by JA, which is an important defense-related phytohormone implicated in the regulation of plant resistance to pathogens. In order to determine the function of ATAF1 in biotic stress reactions, we checked the disease susceptibility of the different groups of plants inoculated with $B$. cinerea, a nonspecific necrotrophic pathogen. Leaves from 4-week-old ATAF1-O1 and ATAF1-O2 plants, ataf1-1 and ataf1-2 mutants, and wild-type plants were drop-inoculated with the $B$. cinerea spore suspension, and disease progression was observed 7 days after inoculation. The examined disease symptoms included chlorosis, necrosis expansion, and tissue maceration surrounding the primary infection 
sites. As shown in Figure 6A, ATAF1-O plants were more sensitive to $B$. cinerea infection in detached leaves than in atafl mutants and wild-type plants, in which B.cinerea infection resulted in milder disease symptoms. Furthermore, we found that the leaf lesion size of ATAF1-O plants was larger than that of other plants (by 9- to 16fold) (Figure 6B).

To define whether the role of ATAF1 in biotic responses of plants was restricted in their reaction to a necrotrophic pathogen, we also tested its function by using the hemi-biotrophic pathogen Pseudomonas syringae. Both ataf1-1 mutant and ATAF1-O1 plants showed comparable disease symptoms in response to the avirulent bacterial strain ( $P$. syringae pv tomato avirulent strain DC3000 carrying avrRpml) and the virulent strain DC3000 in comparison with wild-type plants (Supplementary information, Figure S5).

We examined the expression of the defense-response markers PDF1.2 (linked to JA and ET signalings), and PR1 (linked to systemic acquired resistance (SAR)). As shown in Figure 6C, PDF1.2 expression pattern was unaffected in the ataf1-1 mutant and ATAF1-O1 plants in response to $B$. cinerea, suggesting that the ATAF1 gene functions via a resistance/response pathway that may be independent of PDF1.2. However, ATAF1-O1 plants induced $P R 1$ expression more strongly than other plants, and the lowest expression of $P R 1$ was found in ataf1-1 mutants (Figure 6C), revealing a negative correlation between $A T A F 1$ and plant disease resistance.

Transgenic plants overexpressing ATAF1 are hypersensitive to oxidative stress

Reactive oxygen intermediates (ROIs) have been implicated in signaling in response to both pathogens and abiotic stresses. In plant abiotic stresses, ROIs likely act as signals to induce reactive oxygen scavengers and other protective mechanisms, and as damaging agents that may contribute to stress injury in plants. Additionally, ROIs play important roles in plant-pathogen interactions such as cell wall strengthening and rapid host cell death. To test whether ATAF1 was involved in ROI signaling, the sensitivity of plants to oxidative stress was assayed using rose bengal that generates ROIs in medium when exposed to light, and paraquat (methyl viologen) that increases the ROI formation by inhibiting electron transport in the reduction of NADP to NADPH during photosynthesis [13]. In the presence of $6 \mu \mathrm{M}$ rose bengal, a complete block was found in the growth of ATAF1-O1 and ATAF1-O2 seedlings that were etiolated without any expanded green leaves (Figure 7). However, ataf1-1, ataf1-2, and control plants were minimally affected by this treatment, as these plants developed normally. Like-

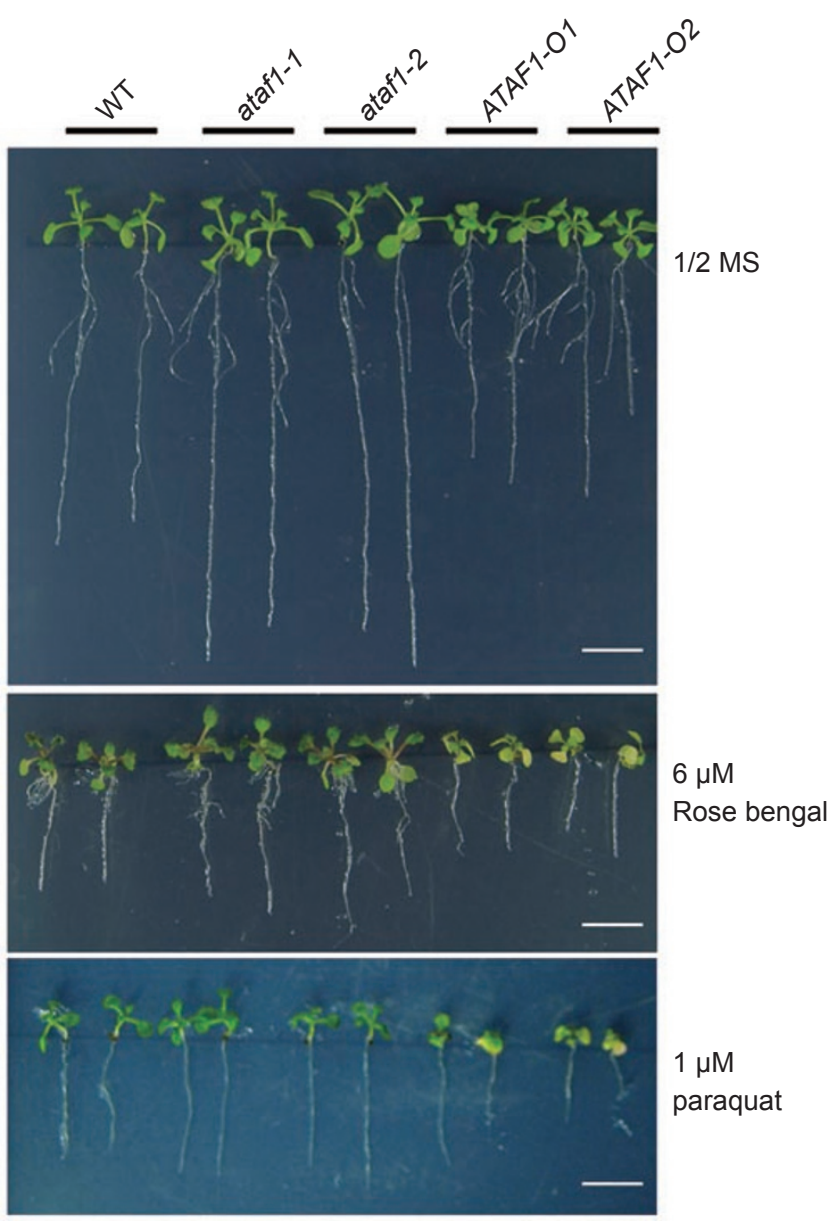

Figure 7 ATAF1-overexpressing plants are hypersensitive to oxidative stresses. Seedlings (5-day old) germinated on 1/2 MS medium were transferred to $1 / 2 \mathrm{MS}$ medium with or without $6 \mu \mathrm{M}$ rose bengal. Photographs were taken 5 days after treatment (middle panel). Young seedlings were compared between wildtype, ataf1-1, ataf1-2, ATAF1-O1, and ATAF1-O2 plants in the presence of $1 \mu \mathrm{M}$ paraquat (lower panel). The pictures were taken after 3 weeks. Bar $=1 \mathrm{~cm}$.

wise, ATAF1-O1 and ATAF1-O2 seedlings showed more sensitivity than other seedlings when germinated on the $1 / 2 \mathrm{MS}$ medium supplemented with $1 \mu \mathrm{M}$ paraquat (Figure 7). These results suggest that ATAF1-mediated stress responses are likely related to ROI signaling pathways.

\section{Discussion}

ATAF1 functions in ABA signaling and abiotic stress responses

ABA is an essential mediator in triggering plant responses to most of the common abiotic stresses, including drought, salinity, high temperature, oxidative stress, and cold $[35,36]$. Nearly all ABA-deficient $(a b a)$ and 
ABA-insensitive (abi) mutants exhibit salt insensitivity during germination and are susceptible to drought because of the impaired stomatal aperture regulation (e.g., abil and abi2), while plants overexpressing the ABA-response pathway genes, such as $S D I R 1, A B F 3$, and $A B F 4$, are tolerant to drought and hypersensitive to salt $[33,34$, 37, 38]. ATAF1 overexpression plants showed remarkably enhanced tolerance to drought but were hypersensitive to salt stress.

Stomatal closure is a key ABA-controlled process in dealing with water deficit conditions. Our results indicate that ATAF1 is involved in stomatal regulation. Its overexpression resulted in lower transpiration and enhanced drought tolerance. The stomatal openings of ATAF1-O1 transgenic plants were smaller than those of wild-type plants under normal light growth conditions in soil. Furthermore, stomatal closure in the epidermis of ATAF1-O1 plants was more sensitive than that of wildtype and ataf1-1 plants after ABA treatment. ATAF1-O1 plants also exhibited the most severe growth inhibition by ABA. Thus, our results suggest that ATAF1 may play a positive role in ABA-mediated guard cell control and regulation of drought response.

Recently, ATAF1 was described as a negative regulator in plant drought tolerance by phenotypically comparing wild-type plants and ataf1 mutants [32]. However, here we found the opposite phenotypes of drought response. We have carefully read the previous paper and compared our experiments. We used the same Salk mutants to validate the documented function of ATAF1. Very surprisingly, we could not observe the same phenotype for these mutant lines as what was previously reported. In fact, we repeatedly observed that $A T A F 1$ overexpression lines, but not mutant plants, were more tolerant to soil-drought stress than controls. Such findings were also supported by other physiological experiments, including leaf water loss measurement and stomatal closure assay. Moreover, we found more rapid induction of some marker genes in the mutant plants compared with other assayed plants under drought stress, but the induction was dramatically diminished at late stage of the treatment. In contrast, ATAF1-O1 plants had significant transcript accumulations of some stress-responsive markers at the late time point, demonstrating a positive correlation with the enhanced drought tolerance of these plants. Considering that the plant response to drought stress spans a relatively long time, high accumulation of stress-responsive genes at late stage of the stress may be a major contributor to plant drought tolerance.

\section{ATAF1 functions in biotic stress}

In this study, ATAF1 enhanced susceptibility to in- fection by $B$. cinerea, but did not influence the plant response to $P$. syringae pv tomato. SAR is a mechanism of induced defense that confers long-lasting protection against a broad spectrum of microorganisms. Pathogentriggered SAR process is dependent on the phytohormone SA. In contrast, plant pathogenesis caused by necrotrophic pathogens is closely related to JA and ET defense pathways [39]. ATAF1 was found to rapidly respond to induction by MeJA, but not by SA (data not shown). To date, molecular and cellular mechanisms in plant-necrotrophic pathogen interactions remain largely elusive. However, ROIs are considered to be important molecules involved in plant disease reaction to necrophrophic pathogens such as $B$. cinerea. Elevated ROI levels can promote hypersensitive cell death. In one previous study, ROIs were reported to be induced by $B$. cinerea to accumulate to a high level, which may be toxic to host plants and consequently facilitate infection [40]. Conversely, reciprocal experiments indicated that chemical-mediated blockade of ROI generation decreased cell death in different plants challenged by pathogens [41, 42] and restricted necrosis development in B. cinereainfected Arabidopsis [43]. The disease assay in our study showed that ATAF1-O plants displayed enhanced lesion formation after $B$. cinerea infection, indicating the negative role of ATAF1 in plant defense to this necrotrophic fungus. Alternatively, ATAF1 overexpression rendered plants hypersensitive to both the rose bengal and paraquat compounds, which promote the generation of reactive oxygen species. Considering the important roles of ROIs in necrotrophic disease processes, these findings suggest that ATAF1 may sensitize the reaction of plant cells to ROIs and accordingly contribute to the enhancement of plant susceptibility after $B$. cinerea infection. In our study, more induction of $P R 1$ transcripts was found in ATAF1-O1 plants infected with $B$. cinerea, implying that there was a $B$. cinerea-triggered hypersensitive response that was thought to positively correlate with $B$. cinerea growth [43]. In addition to pathogen challenge, abiotic stresses such as high salinity can elevate ROI levels in plants. The salt-hypersensitivity of ATAF1-O plants may also be a consequence of their enhanced sensitivity to ROIs.

Functional overlap between ATAF1 and other stressregulatory $N A C$ genes

In this study, mutant lines did not show distinct morphological variations as well as stress-related phenotypic changes when compared with control plants. Therefore, we speculate that there is likely functional redundancy among the NAC genes. Many Arabidopsis NAC members have been predicted to be stress-signaling related 
(Table S1). Recent studies have demonstrated that several $N A C$ genes were involved in plant abiotic stress responses, including RD26 and its homologs ANACO19 and $A N A C 055$. These $N A C$ genes are responsive to drought and high salinity, as well as plant hormones ABA and JA, with varied expression patterns. Overexpression of these $N A C$ genes in plants increased the survival rate for plants grown in water-deficient soil [25]. In this study, ATAF 1 overexpression resulted in many stress-related physiological effects in plants. These effects were similar to the observations described for RD26, ANAC019, and ANAC055. ATAF1 functionally resembles these characterized $N A C$ genes, especially $R D 26$. For example, both $A T A F 1$ and RD26 were induced by exogenous ABA and by drought or high salinity in an ABA-independent manner, and their overexpression transgenic plants showed similar hypersensitivities to ABA. Obviously, functional overlap in stress responses exists among these $N A C$ genes. The functional redundancy is likely responsible for the almost unchanged phenotype in the atafl mutant lines.

In spite of the extensive functional similarities between $A T A F 1$ and other $N A C$ homologs, the function of $A T A F 1$ is not completely identical to that of other $N A C$ genes, including RD26. For example, transcript accumulation was observed for $R D 26$ in response to cold stress [44], but this was not found with ATAF1 (data not shown). Recently, OSNAC6, a rice ortholog of ATAF1, was reported to be a positive contributor to disease resistance and salinity tolerance $[29,30]$. This finding may indicate the functional discrepancies of $N A C$ genes between monocotyledon and dicotyledon plants. In the biotic stress assay, we showed that ATAF1 played a negative role in plant response to $B$. cinerea, resembling the function of $A T A F 2$ in plant pathogenesis. ATAF2, the closest Arabidopsis homolog of ATAF1, has been described as a repressor, which downregulated many pathogenesis-related genes, and consequently enhanced plant susceptibility to Fusarium oxysporum (a soil-borne fungal pathogen) in $A T A F 2$ overexpression lines. Both ATAF 1 and ATAF 2 can be induced by the wounding/ pathogenesis-related phytohormone MeJA. Moreover, we repeatedly isolated ATAF2 as a protein partner of ATAF1 in yeast two-hybrid experiments (unpublished data). These findings suggest that these two proteins are functionally associated and redundant. Nevertheless, it seems that both $N A C$ genes also maintain their unique features. For example, unlike ATAF1, ATAF2 cannot be induced by ABA [28]. Taken together, many other $N A C$ homologs could contribute to the plant abiotic and biotic stress responses. Plants have evolved multiple functionally related NAC proteins that may be beneficial for their survival, given the fact that they are frequently challenged by extreme circumstances.

\section{ATAF1 is an important gene with diverse biological func-} tions

Based on our experimental data, ATAF 1-O transgenic lines showed many evident growth phenotypic changes such as delayed flowering time and sterility at early flowering stage. As a regulator in ABA signaling, ATAF1 can activate $\mathrm{ABA}$-responsive gene expression and result in ABA sensitivity. Similarly, other ABA signaling-related transcription factors such as $\mathrm{ABF} 4$ and $\mathrm{ABI} 3$ were also described to produce the phenotype of delayed flowering time in their overexpression lines [34, 45]. Thus, we hypothesize that the ABA signaling pathway transduced by ATAF1 is likely involved in the flowering process. However, the intrinsic molecular link between ATAF1 and flowering control requires further elucidation.

In conclusion, ATAF1 appears to be a multiple-function transcription factor not only participating in abiotic and biotic stress responses, but also potentially regulating plant developmental processes.

\section{Materials and Methods}

\section{Plant material and growth conditions}

Arabidopsis accession Columbia (Col-0) was used for ATAFI cDNA cloning, gene transformation, and phenotypic control in this study. Salk067648 and Salk057618 (ATAF1 T-DNA insertion mutants), as well as aba2, were obtained from the Arabidopsis Biological Resource Center. Plant growth conditions were as described previously [17].

\section{Gene expression analysis}

Seedlings (3-week old) were used for the following treatments. Dehydration treatment was carried out at room temperature with $75 \%$ humility for seedlings harvested from MS plates and placed on filter paper. Other treatments were carried out for seedlings grown on MS with $250 \mathrm{mM} \mathrm{NaCl}, 50 \mu \mathrm{M} \mathrm{ABA}$, and $50 \mu \mathrm{M}$ MeJA. For gene expression analysis, total RNA was isolated from nitrogen-frozen seedlings using the hot-phenol method [21]. Totally, $15 \mu \mathrm{g}$ RNA was applied in each lane in RNA gel analysis. Hybridizations were performed with the $\alpha^{-32}$ p-labeled ATAF1specific probe derived from its $\mathrm{C}$-terminal region.

\section{Constructs for plant transformation}

To create the transgenic construct for overexpressing ATAF1 in Arabidopsis, the cDNA was amplified by RT-PCR with the primers (5'-GGATCC TAA TGT CAG AAT TAT TAC AGT TG$3^{\prime}$ and $5^{\prime}$-ACTAGT CTA GTA AGG CTT CTG CAT G-3'), and cloned into the site of BamHI and SpeI in the vector pBA002Myc. In this construct, $\mathrm{N}$-terminal $A T A F 1$ was fused in frame to the $\mathrm{C}$-terminal $6 \times M y c$ tag and the tagged protein was driven by the CaMV $35 S$ promoter. The transformants were selected by screening seedlings with resistance against Basta $(8 \mu \mathrm{g} / \mathrm{ml})$ on an MS plate. Myc-ATAF1 expression level was checked by RT-PCR reac- 
tion and western blot with an anti-Myc monoclonal antibody.

\section{Abiotic stress assays}

Seeds were sterilized and suspended in $0.15 \%$ agar and plated on MS medium (including vitamins, M0222.0050, Duchefa Biochemie. B.V., The Netherlands) supplemented with $1.5 \%$ sucrose, then stratified in darkness at $4{ }^{\circ} \mathrm{C}$ for 3 days and transferred to growth chambers with the same environmental conditions described above. To conduct germination rate assay, seeds were plated on MS medium without or with $\mathrm{ABA}(0 \mu \mathrm{M}, 0.25 \mu \mathrm{M}, 0.5 \mu \mathrm{M}$, and $1 \mu \mathrm{M}), \mathrm{NaCl}(50 \mathrm{mM}, 100 \mathrm{mM}$, and $150 \mathrm{mM})$, or $1 \mu \mathrm{M}$ paraquat. To conduct root growth assay, seeds sown on MS plates were stratified for 3 days at $4{ }^{\circ} \mathrm{C}$ and were vertically grown for 4 days under normal conditions, and then seedlings were transferred to vertical square MS plates with or without $10 \mu \mathrm{M}$ ABA. For drought stress, 10-day-old seedlings were transferred from MS plates to water-saturated soil for 7 days, and then water was withheld until plants showed evident drought-stressed phenotypes. For leaf water loss assay, rosette leaves excised from plants grown in soil were put on filter paper for air-dry treatment. The leaf weight was measured at a series of time points. For rose bengal treatment, 5-day-old seedlings germinated on $1 / 2$ MS medium were transferred to $1 / 2$ medium with $6 \mu \mathrm{M}$ rose bengal.

Effects of ABA on stomatal aperture of plants. Epidermal peels from 5-week-old plants were kept for $12 \mathrm{~h}$ in the dark, incubated under light in buffer $(10 \mathrm{mM} \mathrm{KCl}, 7.5 \mathrm{mM}$ potassium iminodiacetate, and $10 \mathrm{mM}$ MES-KOH, $\mathrm{pH} 6.15$ ) at $20{ }^{\circ} \mathrm{C}$ for $3 \mathrm{~h}$, and then treated with 0 and $50 \mu \mathrm{M}$ ABA for $2 \mathrm{~h}$ before aperture measurements (measured with a ZEISS microscope).

\section{Fungal culture and plant inoculation}

$B$. cinerea was grown on potato dextrose agar for 7-10 days at $24{ }^{\circ} \mathrm{C}$ with a $12-\mathrm{h}$ photoperiod before spore collection. Spores were collected by flooding the dish and filtering the suspension with gauze. The conidia were resuspended with potato dextrose broth, and the concentration was adjusted to $10^{5}$ spores $/ \mathrm{ml}$. Whole plants or rosette leaves were placed in dishes containing $0.8 \%$ agar and inoculations were performed with a $3 \mu 1$ droplet of $B$. cinerea spores. After inoculation, plants were kept under a transparent cover to maintain high humidity and transferred to a growth chamber with $21^{\circ} \mathrm{C}$ day and $18{ }^{\circ} \mathrm{C}$ night temperatures.

\section{Acknowledgments}

We would like to thank Dr Nam-Hai Chua (Rockefeller University) for kindly providing the pBA002Myc vector and the Arabidopsis Biological Resource Center (ABRC), Ohio State University for providing T-DNA insertion lines. This work was supported by grants from National Natural Science Foundation of China (No. 30530400/90717006/30670195) to Q Xie and Y Wu, the Chinese Academy of Science (KSCX2-YW-N-010 and CXTD-S2005-2), and the Guangdong Natural Science Foundation, China (No. 5300648) to Z Deng.

\section{References}

1 Torres MA, Dangl JL. Functions of the respiratory burst oxidase in biotic interactions, abiotic stress and development.
Curr Opin Plant Biol 2005; 8:397-403.

2 Apel K, Hirt H. Reactive oxygen species: metabolism, oxidative stress, and signal transduction. Annu Rev Plant Biol 2004; 55:373-399.

3 Mauch-Mani B, Mauch F. The role of abscisic acid in plantpathogen interactions. Curr Opin Plant Biol 2005; 8:409-414.

4 Fujita M, Fujita Y, Noutoshi Y, et al. Crosstalk between abiotic and biotic stress responses: a current view from the points of convergence in the stress signaling networks. Curr Opin Plant Biol 2006; 9:436-442.

5 Seki M, Narusaka M, Ishida J, et al. Monitoring the expression profiles of 7000 Arabidopsis genes under drought, cold and high-salinity stresses using a full-length cDNA microarray. Plant J 2002; 31:279-292.

6 AbuQamar S, Chen X, Dhawan R, et al. Expression profiling and mutant analysis reveals complex regulatory networks involved in Arabidopsis response to Botrytis infection. Plant $J$ 2006; 48:28-44.

7 Cheong YH, Chang HS, Gupta R, et al. Transcriptional profiling reveals novel interactions between wounding, pathogen, abiotic stress, and hormonal responses in Arabidopsis. Plant Physiol 2002; 129:661-677.

8 Davletova S, Schlauch K, Coutu J, Mittler R. The zinc-finger protein Zat12 plays a central role in reactive oxygen and abiotic stress signaling in Arabidopsis. Plant Physiol 2005; 139:847-856.

9 Schenk PM, Kazan K, Wilson I, et al. Coordinated plant defense responses in Arabidopsis revealed by microarray analysis. Proc Natl Acad Sci USA 2000; 97:11655-11660.

10 Dombrecht B, Xue GP, Sprague SJ, et al. MYC2 differentially modulates diverse jasmonate-dependent functions in Arabidopsis. Plant Cell 2007; 19:2225-2245.

11 Laurie-Berry N, Joardar V, Street IH, Kunkel BN. The Arabidopsis thaliana JASMONATE INSENSITIVE 1 gene is required for suppression of salicylic acid-dependent defenses during infection by Pseudomonas syringae. Mol Plant Microbe Interact 2006; 19:789-800.

12 Lorenzo O, Chico JM, Sanchez-Serrano JJ, Solano R. JASMONATE-INSENSITIVE1 encodes a MYC transcription factor essential to discriminate between different jasmonateregulated defense responses in Arabidopsis. Plant Cell 2004; 16:1938-1950.

13 Mengiste T, Chen X, Salmeron J, Dietrich R. The BOTRYTIS SUSCEPTIBLE1 gene encodes an R2R3MYB transcription factor protein that is required for biotic and abiotic stress responses in Arabidopsis. Plant Cell 2003; 15:2551-2565.

14 Hibara K, Takada S, Tasaka M. CUC1 gene activates the expression of SAM-related genes to induce adventitious shoot formation. Plant J 2003; 36:687-696.

$15 \mathrm{He}$ XJ, Mu RL, Cao WH, et al. AtNAC2, a transcription factor downstream of ethylene and auxin signaling pathways, is involved in salt stress response and lateral root development. Plant J 2005; 44:903-916.

16 Ishida T, Aida M, Takada S, Tasaka M. Involvement of CUPSHAPED COTYLEDON genes in gynoecium and ovule development in Arabidopsis thaliana. Plant Cell Physiol 2000; 41:60-67.

17 Xie Q, Frugis G, Colgan D, Chua NH. Arabidopsis NAC1 transduces auxin signal downstream of TIR1 to promote lat- 
eral root development. Genes Dev 2000; 14:3024-3036.

18 Guo Y, Gan S. AtNAP, a NAC family transcription factor, has an important role in leaf senescence. Plant J 2006; 46:601612.

19 Collinge M, Boller T. Differential induction of two potato genes, Stprx2 and StNAC, in response to infection by Phytophthora infestans and to wounding. Plant Mol Biol 2001; 46:521-529.

20 Selth LA, Dogra SC, Rasheed MS, et al. A NAC domain protein interacts with tomato leaf curl virus replication accessory protein and enhances viral replication. Plant Cell 2005; 17:311-325

21 Xie Q, Sanz-Burgos AP, Guo H, Garcia JA, Gutierrez C. GRAB proteins, novel members of the NAC domain family, isolated by their interaction with a geminivirus protein. Plant Mol Biol 1999; 39:647-656.

22 Jensen MK, Rung JH, Gregersen PL, et al. The HvNAC6 transcription factor: a positive regulator of penetration resistance in barley and Arabidopsis. Plant Mol Biol 2007; 65:137150.

23 Nakashima K, Kiyosue T, Yamaguchi-Shinozaki K, Shinozaki K. A nuclear gene, erd1, encoding a chloroplast-targeted Clp protease regulatory subunit homolog is not only induced by water stress but also developmentally up-regulated during senescence in Arabidopsis thaliana. Plant J 1997; 12:851-861.

24 Simpson SD, Nakashima K, Narusaka Y, et al. Two different novel cis-acting elements of erd1, a clpA homologous Arabidopsis gene function in induction by dehydration stress and dark-induced senescence. Plant J 2003; 33:259-270.

25 Tran LS, Nakashima K, Sakuma Y, et al. Isolation and functional analysis of Arabidopsis stress-inducible NAC transcription factors that bind to a drought-responsive cis-element in the early responsive to dehydration stress 1 promoter. Plant Cell 2004; 16:2481-2498.

26 Zheng X, Chen B, Lu G, Han B. Overexpression of a NAC transcription factor enhances rice drought and salt tolerance. Biochem Biophys Res Commun 2009; 379:985-989.

$27 \mathrm{Bu}$ Q, Jiang H, Li CB, et al. Role of the Arabidopsis thaliana NAC transcription factors ANAC019 and ANAC055 in regulating jasmonic acid-signaled defense responses. Cell Res 2008; 18:756-767.

28 Delessert C, Kazan K, Wilson IW, et al. The transcription factor ATAF2 represses the expression of pathogenesis-related genes in Arabidopsis. Plant J 2005; 43:745-757.

29 Nakashima K, Tran LS, Van ND, et al. Functional analysis of a NAC-type transcription factor OsNAC6 involved in abiotic and biotic stress-responsive gene expression in rice. Plant $J$ 2007; 51:617-630.

30 Ohnishi T, Sugahara S, Yamada T, et al. OsNAC6, a member of the NAC gene family, is induced by various stresses in rice. Genes Genet Syst 2005; 80:135-139.

31 Jensen MK, Hagedorn PH, de Torres-Zabala M, et al. Tran- scriptional regulation by an NAC (NAM-ATAF1,2-CUC2) transcription factor attenuates ABA signaling for efficient basal defence towards Blumeria graminis f. sp. hordei in Arabidopsis. Plant J 2008; 56:867-880.

32 Lu PL, Chen NZ, An R, et al. A novel drought-inducible gene, ATAF1, encodes a NAC family protein that negatively regulates the expression of stress-responsive genes in Arabidopsis. Plant Mol Biol 2007; 63:289-305.

33 Zhang Y, Yang C, Li Y, et al. SDIR1 is a RING finger E3 ligase that positively regulates stress-responsive abscisic acid signaling in Arabidopsis. Plant Cell 2007; 19:1912-1929.

34 Kang JY, Choi HI, Im MY, Kim SY. Arabidopsis basic leucine zipper proteins that mediate stress-responsive abscisic acid signaling. Plant Cell 2002; 14:343-357.

35 Finkelstein RR, Lynch TJ. The Arabidopsis abscisic acid response gene ABI5 encodes a basic leucine zipper transcription factor. Plant Cell 2000; 12:599-609.

$36 \mathrm{Hu} \mathrm{H}$, Dai M, Yao J, et al. Overexpressing a NAM, ATAF, and CUC (NAC) transcription factor enhances drought resistance and salt tolerance in rice. Proc Natl Acad Sci USA 2006; 103:12987-12992.

37 Leon-Kloosterziel KM, Gil MA, Ruijs GJ, et al. Isolation and characterization of abscisic acid-deficient Arabidopsis mutants at two new loci. Plant J 1996; 10:655-661.

38 Murata Y, Pei ZM, Mori IC, Schroeder J. Abscisic acid activation of plasma membrane $\mathrm{Ca}\left({ }^{2+}\right)$ channels in guard cells requires cytosolic $\mathrm{NAD}(\mathrm{P}) \mathrm{H}$ and is differentially disrupted upstream and downstream of reactive oxygen species production in abi1-1 and abi2-1 protein phosphatase 2C mutants. Plant Cell 2001; 13:2513-2523.

39 Glazebrook J. Contrasting mechanisms of defense against biotrophic and necrotrophic pathogens. Annu Rev Phyto pathol 2005; 43:205-227.

40 Mittler R. Oxidative stress, antioxidants and stress tolerance. Trends Plant Sci 2002; 7:405-410.

41 Kazan K, Murray FR, Goulter KC, Llewellyn DJ, Manners JM. Induction of cell death in transgenic plants expressing a fungal glucose oxidase. Mol Plant Microbe Interact 1998; 11:555-562.

42 Naton B, Hahlbrock K, Schmelzer E. Correlation of rapid cell death with metabolic changes in fungus-infected, cultured parsley cells. Plant Physiol 1996; 112:433-444.

43 Govrin EM, Levine A. The hypersensitive response facilitates plant infection by the necrotrophic pathogen Botrytis cinerea. Curr Biol 2000; 10:751-757.

44 Fujita M, Fujita Y, Maruyama K, et al. A dehydration-induced NAC protein, RD26, is involved in a novel ABA-dependent stress-signaling pathway. Plant $J$ 2004; 39:863-876.

45 Zhang X, Garreton V, Chua NH. The AIP2 E3 ligase acts as a novel negative regulator of ABA signaling by promoting ABI3 degradation. Genes Dev 2005; 19:1532-1543.

(Supplementary information is linked to the online version of the paper on the Cell Research website.) 